\title{
Long-term administration of traditional kampo medicine shimotsuto, juzentaihoto and unseiin inhibits experimental thrombosis in mice
}

\author{
Yoshinobu Ijiri $^{1,2}$, Hiroko Anzai ${ }^{3}$, Weifua $\mathrm{Gao}^{4}$, Kunio Takahashi ${ }^{5}$, Naemi Kajiwara ${ }^{3}$, \\ Masahiro Murakami ${ }^{2,4}$, Junichiro Yamamoto ${ }^{2,6 *}$ \\ ${ }^{1}$ Department of Food Nutritional Science, Faculty of Liberal Arts, Osaka Shoin Women's University, Osaka, Japan \\ ${ }^{2}$ Antithrombotic Diet Discussion Group, Laboratory of Physiology, Faculty of Nutrition, Kobe Gakuin University, Kobe, Japan \\ ${ }^{3}$ Laboratory of Nutrition Physiology, Faculty of Health and Welfare, Kobe Women's University, Kobe, Japan \\ ${ }^{4}$ Laboratory of Pharmaceutics, Faculty of Pharmacy, Osaka-ohtani University, Osaka, Japan \\ ${ }^{5}$ Zaiseido Pharmaceutical Co Ltd, Wakayama, Japan \\ ${ }^{6}$ Laboratory of Physiology, Faculty of Nutrition and Cooperative Research Center of Life Sciences, Kobe Gakuin University, Kobe, \\ Japan; *Corresponding Author: yamamoto@nutr.kobegakuin.ac.jp
}

Received 13 January 2010; revised 27 January 2010; accepted 30 January 2010.

\begin{abstract}
Traditional Chinese herbal medicines (Kampo) are used to improve flow characteristics of blood (Oketsu). We assumed that by preventing stagnation of blood, these medicines may be beneficial not only in venous but in arterial thrombotic conditions. The present study aimed to assess the antithrombotic effect of three Kampo, using well-established in vitro and animal models of thrombosis. Western-style highfat diet containing 1\% Kampo (Shimotsuto, Juzentaihoto or Unseiin) was administered to C57BL/6 mice for 12 weeks. The effect on thrombus formation by laser irradiation of the carotid artery of mice was assessed. In addition the ex-vivo technique of shear-induced platelet reactivity measurement (haemostatometry) and the in vivo test of endothelial function (flowmediated vasodilation) were also used to assess the mechanism of antithrombotic effect. All three medicines have significantly inhibited arterial thrombus formation in mice. According to our studies, the mechanism of antithrombotic effect is based on the inhibition of shear-induced platelet reactivity and stimulation of endothelial function (Unseiin). It is assumed that the common ingredients Japanese Angelica Root, Cnidium Rhizome, Peony Root and Rehmannia Root could be responsible for the observed antithrombotic effect.
\end{abstract}

Keywords: Kampo; Chinese Medicine; Thrombosis;
Platelet; Endothelial Function; Stroke; Cardiovascular Disease

\section{INTRODUCTION}

Prevention of lifestyle-related atherothrombotic diseases such as myocardial infarction and stroke is an important and urgent social task in many developed countries. Epidemiological studies have provided evidence for the causative role of inappropriate diet in the development of atherothrombotic diseases and the regular life style and exercise in the prevention of such diseases.

Chinese herbal medicine (Kampo) has a long history of treating various diseases. Currently used Kampo is believed to prevent stagnation thus improve the flow characteristics of blood. In our earlier studies, we used sensitive animal models of arterial thrombosis to assess the actual thrombotic status. We succeeded overcoming the main difficulty of the relative resistance of rodents to the prothrombotic effect of high fat diet. We showed that in apolipoprotein $\mathrm{E}$ and low-density lipoprotein receptor double deficient C57BL/6J mice, high fat diet induced a prothrombotic state, similar to humans [1]. By inducing and measuring the rate of arterial thrombus formation in response to laser irradiation in such spontaneously atherogenic mice, we could test the antithrombotic effect of various substances and diets. In the past, the use of this in vivo test together with other global in vitro thrombosis tests enabled us to find various fruits and vegetables with significant experimental antithrombotic activity $[2,3]$. 


\section{MATERIALS AND METHODS}

\subsection{Chinese Herbal Medicines (Kampo) for Preventing Stagnation of Blood (Oketsu)}

Shimotsuto, Juzentaihoto and Unseiin were used (Table 1). These were supplied by Zaiseido Pharmaceutical Co Ltd, Wakayama, Japan.

\subsection{Diets Containing Kampo}

Experimental diets containing the three Kampos in 1\% (w/w) were prepared by adding Kampo to the Westernstyle high fat model diet (Table 2). Diets were stored at $-30^{\circ} \mathrm{C}$ until use.

\subsection{Animals and Administration of Diet}

Five week old male C57BL/6J mice were purchased from SLC Co Ltd (Hamamatsu, Japan) one week before the experiments and raised for one week on standard solid chow (CE-2, Clea Japan Inc., Tokyo, Japan) and drinking water ad libitum. Subsequently the chow was changed to the experimental diet, starting at age of 6 week, and lasted for 12 weeks. Mice were kept in the Animal Unit of Kobe Women's University, had free access to both diets and water, the room was airconditioned $\left(22.5 \pm 22^{\circ} \mathrm{C}\right.$ and humidity $\left.40-60 \%\right)$ having 12-h light and dark cycle. Animals were fasted overnight before the test and kept in compliance with the "Guiding Principles for the Care and Use of Animals in the field of Physiological Sciences," published by Physiological

Table 1. Composition of the three Kampo for blood stagnancy (Oketsu).

\begin{tabular}{lccc}
\hline \multirow{2}{*}{ Ingredient } & \multicolumn{3}{c}{ Content (g) } \\
\cline { 2 - 4 } & Shimotsuto & Juzentaihoto & Unseiin \\
\hline Japanese Angelica & 4.0 & 3.0 & 4.0 \\
Cnidium Rhizome & 4.0 & 3.0 & 4.0 \\
Peony Root & 4.0 & 3.0 & 4.0 \\
Rehmannia Root & 4.0 & 3.0 & 4.0 \\
Atractylodes Rhizome & 0 & 3.0 & 0 \\
Poria Sclerotium & 0 & 3.0 & 0 \\
Cinnamon Bark & 0 & 3.0 & 0 \\
Ginseng & 0 & 3.0 & 0 \\
Glycyrrhiza & 0 & 1.0 & 0 \\
AStragalus Root & 0 & 3.0 & 0 \\
Coptis Rhizome & 0 & 0 & 1.5 \\
Scutellaria Root & 0 & 0 & 3.0 \\
Phellodendron Bark & 0 & 0 & 1.5 \\
Gardenia Fruit & 0 & 0 & 2.0 \\
\hline
\end{tabular}

Society of Japan. The experiments were approved by the Animal Experiment Committee of Kobe Women's University.

\subsection{He-Ne Laser-Induced Carotid Artery Thrombosis Test}

This technique has been described in detail [1-4]. Briefly, the left femoral artery and the carotid artery $(450-500 \mu \mathrm{M}$ in diameter) of Nembutal anaesthetized mouse were exposed. The mouse was placed on a special microscope stage and through the femoral artery, a heat-absorbing dye Evans blue was injected. Subsequently the centre of the exposed carotid artery was irradiated with $\mathrm{He}-\mathrm{Ne}$ laser. Thrombus formation at the site of irradiation was monitored under epi-illumination and recorded on videotape using CCD camera.

\subsection{Calculation of Thrombus Size}

From the start of laser irradiation, the computer-image of the forming thrombus was recorded in every 10 seconds for 10 minutes. The thrombus mass was delineated and its size was calculated by a software (Image Processing and Analysis Java version 1.30, National Institutes of Health, Bethesda, Maryland, USA). Due to frequent embolisation, the size of thrombus increased (building up the thrombus) or decreased (partial embolisation). Thrombotic status was defined by the total sum of thrombus sizes. An increase of such total sum indicated a prothrombotic state (enhanced thrombus formation), while decrease of such sum indicated an antithrombotic effect.

\subsection{Endothelial Function Test}

The endothelium-dependent flow-mediated vasodilation (FMV) technique originally described for rat was adapted to mice $[5,6]$. The anaesthetized mouse was kept on a heated pad to maintain body temperature; the left femoral artery was exposed, isolated and covered with gauze saturated with $37^{\circ} \mathrm{C}$ saline. Blood flow in the artery was stopped by clamping for 3 minutes and then the flow was restored by releasing the clamp. Diameter of the artery was monitored 2-4 $\mathrm{mm}$ distal from the site of clamping by a CCD camera (Model CS900, Takenaka System Co. Ltd., Kyoto, Japan). Baseline images were taken before clamping and then in every 10 seconds over 60 seconds and further in 30 seconds intervals over 450 seconds after restoration of blood flow. Nitroglycerinmediated vasodilation was induced by placing 70 microliters of $2.2 \mathrm{mM}$ nitroglycerin/saline solution on the artery. The recorded images of the artery were transferred to a computer and the diameter changes were calculated with a software (Image Processing and Analysis; Java version 1.30). Changes in vessel diameter after restoration of flow were expressed as percentage of the baseline values (before clamping or nitroglycerin). 
Table 2. Experimental diets containing Chinese herbal prescriptions (Kampo) for blood stagnancy (Oketsu).

\begin{tabular}{|c|c|c|c|c|}
\hline Ingredient & $\begin{array}{l}\text { Control diet } \\
\text { (High fat diet) }\end{array}$ & Shimotsuto & Juzentaihoto & Unseiin \\
\hline & \multicolumn{4}{|c|}{$g / k g$} \\
\hline Casein & 232 & 232 & 232 & 232 \\
\hline Cystine & 3 & 3 & 3 & 3 \\
\hline Corn starch & 362 & 362 & 362 & 362 \\
\hline Sucrose & 99.5 & 99.5 & 99.5 & 99.5 \\
\hline Soy oil & 30 & 30 & 30 & 30 \\
\hline Butter & 75 & 75 & 75 & 75 \\
\hline Beef tallow & 100 & 100 & 100 & 100 \\
\hline Cellulose & 50 & 50 & 50 & 50 \\
\hline Mineral mix & 35 & 35 & 35 & 35 \\
\hline Vitamin mix & 10 & 10 & 10 & 10 \\
\hline Choline bitartrate & 2.5 & 2.5 & 2.5 & 2.5 \\
\hline tert-Butylhydroquinon & 0.041 & 0.041 & 0.041 & 0.041 \\
\hline Cholesterol & 0.5 & 0.5 & 0.5 & 0.5 \\
\hline Shimotsuto & - & 10 & - & - \\
\hline Juzentaihoto & - & - & 10 & - \\
\hline Unseiin & - & - & - & 10 \\
\hline Energy, $\mathrm{KJ} / 100 \mathrm{~g}$ & 1956 & & & \\
\hline
\end{tabular}

\subsection{Platelet Function Test}

The shear-induced platelet reactivity test (haemostatometry) was performed with a three-channel purposebuilt Haemostatometer constructed in the Physiology Laboratory of the Faculty of Nutrition at Kobe Gakuin University. Details of haemostatometry have been described in detail elsewhere $[7,8]$. In brief, non-anticoagulated blood withdrawn from the abdominal aorta was perfused through polyethylene tubing by oil displacement technique and the perfusion pressure was continuously monitored. When the pressure stabilized at 60 $\mathrm{mmHg}$, through-holes were pierced in the tubing by a fine needle. Escape of blood ("bleeding") through the pierced holes into the surrounding saline resulted in a sharp drop and then a gradual return of the perfusion pressure to the baseline level. The recovery of pressure after the initial drop reflected platelet-rich haemostatic plug formation in the pierced holes. The area of pressure changes (mmHg.s) were calculated ( $\mathrm{H} 1$ and $\mathrm{H} 2)$ and used as an index of platelet reactivity. An increase or decrease of $\mathrm{H} 1$ and $\mathrm{H} 2$ over controls indicated suppressed or enhanced platelet reactivity, respectively. The time from the start of the test until the first decrease of perfusion pressure of at least $10 \mathrm{mmHg}(\mathrm{CT} 1)$ and to a level not higher than $10 \mathrm{mmHg}(\mathrm{CT} 2)$ reflected the initial and completed coagulations, respectively. Prolongation of CT1 and CT2 indicated inhibition of dynamic coagulation, whereas shortening of CT1 and CT2 suggested hypercoagulation.

\subsection{Statistical Analysis}

The effect of Shimotsuto, Juzentaihoto and Unseiin in the applied tests groups was analysed by factorial ANOVA, followed by the post hoc test of Fisher's PLSD using commercially available statistical package Stat View (v. 5.0; SAS Institute Inc., North Carolina, USA). Logarithmic H1, H2 data and FMV data were analyzed by Student's unpaired t-test; CT1 and CT2 values were analyzed without logarithmic conversion. Results were expressed as mean \pm SEM. $\mathrm{P}<0.05$ was considered to be statistically significant.

\section{RESULTS AND DISCUSSIONS}

\subsection{Antithrombotic Effect of Diets Containing Kampos}

Results are shown in Figure 1. Long-term administration 


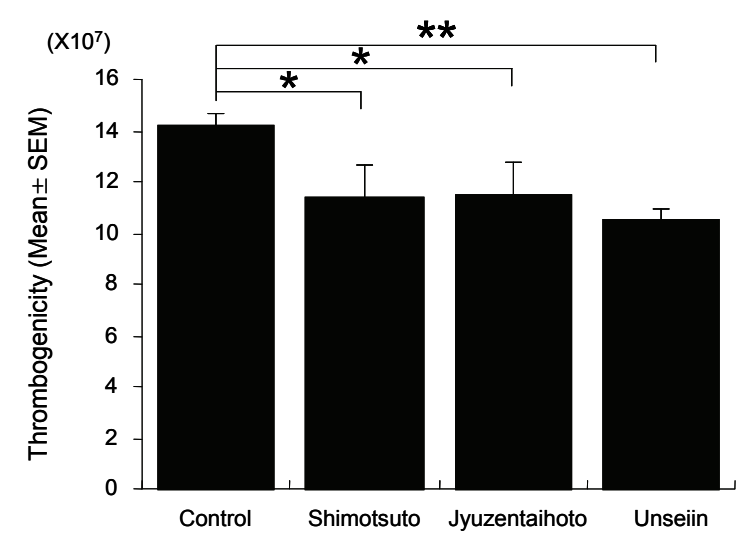

$\mathrm{N}=5-6, * \mathrm{P}<0.05, * * \mathrm{P}<0.01$.

Figure 1. Antithrombotic effect of diets containing various Kampo.

of all Kampo significantly decreased experimental thrombogenicity.

Shimotsuto contains four components common in three antithrombotic Kampos (Table 2): Japanese angelica, Cnidium rhizome, Peony root and Rehmannia root. In contrast, Juzentaihoto and Unseiin contain other additional components than the above common four. These components are added to adjust the efficacy of medicine to individual personality considering the physical and mental characters. The present results show that antithrombotic activity of the tested three Kampos derives from Japanese angelica, Cnidium rhizome, Peony root and Rehmannia root. As Unseiin showed the strongest antithrombotic activity, its effect was further investigated.

\subsection{Effect of Unseiin on Endothelial Function}

The effect of diet containing Unseiin on endothelial function was investigated after 12 weeks feeding by the flow-mediated or endothelium-mediated vasodilation (FMV) and nitroglycerin-mediated vasodilation (NMV) techniques. Results are shown in Figure 2. Endothelium-mediated vasodilation was significantly higher in mice with Unseiin feeding than in the controls but Unseiin did not affect nitroglycerin-induced vasodilation. Thus our finding suggests that Unseiin enhances endothelial function but not medial or muscular layer of the blood vessel.

Keishi-bukuryo-gan (Gui-zhi-fu-ling-wan) has been used for the improvement of blood circulation and recently it is often used to prevent arteriosclerosis. One of the mechanisms involved is thought to be the improvement of endothelial dysfunction [9]. Choto-san has also been shown to improve blood circulation by protecting endothelium [10].

Flow-mediated vasodilation test was developed for di- agnosing arteriosclerosis in humans [11-15] and we have employed this test to animal experiments $[5,6]$. Publications using FMV test are tremendously increasing in humans and demonstrating that nitric oxide and prostacyclin are involved in FMV [16]. Unseiin enhances endothelial function by nitric oxide and prostacyclin generation, and this could be a mechanism of the inhibition of arterial thrombosis.

\subsection{Effect of Unseiin on Shear-Induced Platelet Reactivity}

The diet containing Unseiin was given to mice for 12 weeks and the effect of Unseiin on platelet function was assessed by shear-induced platelet function test (haemostatometry) using non-anticoagulated blood. Results are shown in Figure 3. Unseiin inhibited shear-induced platelet reactivity, but no effect on blood coagulation $[7,8]$.

Kangen-karyu (KGK) is a traditional Chinese herbal medicine to invigorate circulation. It contains 6 herbs, peony root, cnidium rhizome, safflower, cyperus rhizome, saussurea root (JP XIV), and Salvia miltiorrhiza root. KGK significantly extended tail-bleeding time and suppressed exvivo platelet aggregation in mice three days after oral administration, while it did not extend prothrombin time. These findings suggest that the antithrombotic effect of KGK may be due to the inhibition
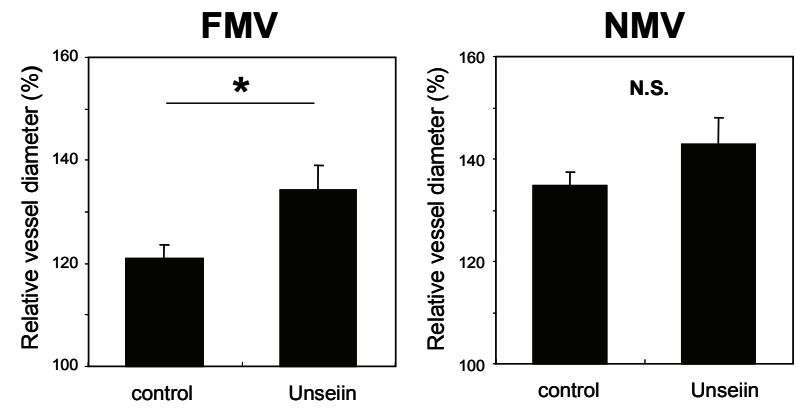

FMV: $\mathrm{n}=8, * \mathrm{P}=0.023$, NMV: $\mathrm{n}=7$, N.S.: not significant $(\mathrm{P}=0.199)$.

Figure 2. Effect of diets containing Unseiin on flow-mediated vasodilation (FMV) and nitroglycerin-mediated vasodilation (NMV).
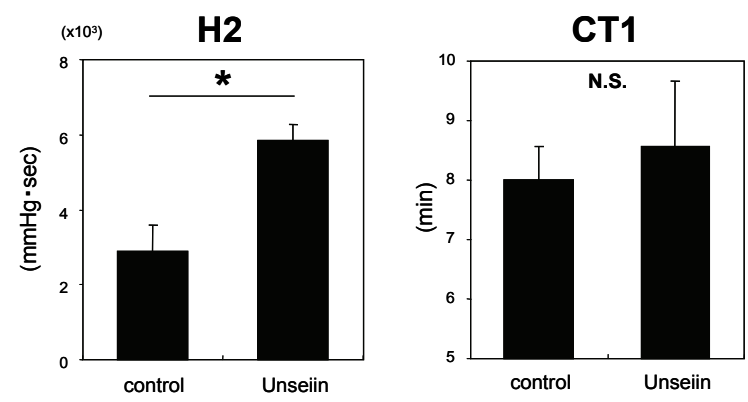

H2: $\mathrm{n}=4, * \mathrm{P}=0.013 ; \mathrm{CT} 1: \mathrm{n}=4$, N.S.: not significant $(\mathrm{P}=0.643)$.

Figure 3. Effect of diets containing Unseiin on shear-induced platelet reactivity and dynamic coagulation. 
of platelet aggregation but not to anticoagulation [17]. The components, peony root and cnidium rhizome are common in KGK and in the currently investigated three Kampos. Peony root and cnidium rhizome might play a major role in antithrombotic activity of Kampo.

Agonist-induced platelet aggregation tests were used to assess of antithrombotic effect of Gosha-jinki-gan [18]. However, there are differences in the effects obtained by agonist-induced and shear-induced platelet function test [19]. We have compared agonist-induced platelet function test using anticoagulated whole blood and shear-induced platelet function test using non-anticoagulated whole blood, and concluded that shear-induced platelet function test using non-anticoagulated whole blood is physiologically more relevant to platelet function existing in vivo.

In conclusion, long-term intake of Shimotsuto, Juzentaihoto and Unseiin inhibited arterial thrombogenesis by their anti-platelet activities and by stimulating the vascular endothelium.

\section{ACKNOWLEDGEMENTS}

We thank Mrs Kinuyo Matsumoto, Ms Miho Hayashi and Ms Ikue Kusuda, Faculty of Health and Welfare, Kobe Women's University for their help of animal care.

\section{REFERENCES}

[1] Ijiri, Y., Miura, M., Hashimoto, M., Fukunaga, C., Watanabe, S., Kubota, A., Oiwa, K., Okuda, T. and Yamamoto, J. (2002) A new model to evaluate the diet-induced prothrombotic state, using He-Ne laser-induced thrombogenesis in the carotid artery of apolipoprotein E-deficient and low-density lipoprotein receptor-deficient mice. Blood Coagul \& Fibrinolysis, 13(6), 497-504.

[2] Yamamoto, J., Taka, T., Yamada, K., Ijiri, Y., Murakami, M., Hirata, Y., Naemura, A., Hashimoto, M., Yamashita, T., Oiwa, K., Seki, J., Suganuma, H., Inakuma, T. and Yoshida, T. (2003) Tomatoes have natural anti-thrombotic effects. British Journal of Nutrition, 90(6), 10311038.

[3] Yamamoto, J., Naemura, A., Ijiri, Y., Ogawa, K., Suzuki, T., Shimada, Y. and Giddings, J.C. (2008) The antithrombotic effects of carrot filtrates in rats and mice. Blood Coagul \& Fibrinolysis, 19(8), 785-792.

[4] Kovacs, I.B., Tigyi-Sebes, A., Trombitas, K. and Gorog, P. (1975) Evans blue: An ideal energy-absorbing material to produce intravascular microinjury by $\mathrm{He}-\mathrm{Ne}$ gas laser. Microvascular Research, 10, 107-124.

[5] Taka, T., Yoshio, Y., Seki, J., Giddings, J.C. and Yamamoto, J. (2002) Impaired flow-mediated vasodilation in vivo and reduced shear-induced platelet reactivity in vitro in response to nitric oxide in prothrombotic, strokeprone spontaneously hypertensive rats. Pathophysiology of Haemostasis \& Thrombosis, 32, 184-189.

[6] Tamura, Y., Naemura, A., Inoue, A., Ijiri, Y., Seki, J., Yada, T., Goto, M., Shinohara, M., Kawashima, S., Gid- dings, J.C. and Yamamoto, J. (2009) Impaired endothelial function may be due to decreased aortic tetrahydrobiopterin, assessed by a new flow-mediated vasodilation in vivo in hypercholesterolemic/atherogenic mice. Blood Coagul \& Fibrinolysis, 20(8), 699-705.

[7] Ratnatunga, C.P., Edmondson, S.F., Rees, G.M. and Kovacs, I.B. (1992) High-dose aspirin inhibits shear-induced platelet reaction involving thrombin generation. Circulation, 85(3), 1077-1082.

[8] Yamamoto, J., Taka, T., Nakajima, S., Ueda, M., Sugimoto, E., Sasaki, Y., Muraki, T., Seki, J. and Watanabe, S. (1999) A shear-induced in vitro platelet function test can assess clinically relevant anti-thrombotic effects. Platelets, 10(2), 178-184.

[9] Goto, H., Shimada, Y., Sekiya, N., Yang, Q., Kogure, T., Mantani, N., Hikiami, H., Shibahara, N. and Terasawa, K. (2004) Effects of keishi-bukuryo-gan on vascular function and hemorheological factors in spontaneously diabetic (WBN/kob) rats. Phytomedicine, 11(2-3), 188-195.

[10] Yang, Q., Goto, H., Shimada, Y., Kita, T., Shibahara, N. and Terasawa, K. (2002) Effects of choto-san on hemorheological factors and vascular function in stroke-prone spontaneously hypertensive rats. Phytomedicine, 9, 93-98.

[11] Moens, A.A., Goovaerts, I., Claeys, M.J. and Vrints, C.J. (2005) Flow-mediated vasodilation: A diagnostic instrument, or an experimental tool? Chest, 127(6), 2254-2263.

[12] Soga, J., Nishioka, K., Nakamura, S., Umemura, T., Jitsuiki, D., Hidaka, T., Teragawa, H., Takemoto, H., Goto, C., Yoshizumi, M., Chayama, K. and Higashi, Y. (2007) Measurement of flow-mediated vasodilation of the brachial artery: A comparison of measurements in the seated and supine positions. Circulation Journal, 71(5), 736-740.

[13] Matsuo, S., Matsumoto, T., Takashima, H., Ohira, N., Yamane, T., Yasuda, Y., Tarutani, Y. and Horie, M. (2004) The relationship between flow-mediated brachial artery vasodilation and coronary vasomotor responses to bradykinin: Comparison with those to acetylcholine. Journal of Cardiovascular Pharmacology, 44(2), 164170.

[14] Tarutani, Y., Matsumoto, T., Takashima, H., Yamane, T. and Horie, M. (2005) Brachial artery flow-mediated vasodilation is correlated with coronary vasomotor and fibrinolytic responses induced by bradykinin. Hypertension Research, 28(1), 59-66.

[15] Anderson, T.J., Uehata, A., Gerhard, M.D., Meredith, I.T., Knab, S., Delagrange, D., Lieberman, E.H., Ganz, P., Creager, M.A., Yeung, A.C. and Selwyn, A.P. (1995) Close relation of endothelial function in the human coronary and peripheral circulations. Journal of the American College of Cardiology, 26(5), 1235-1241.

[16] Engelke, K.A., Halliwill, J.R., Proctor, D.N., Dietz, N.M. and Joyner, M.J. (1996) Contribution of nitric oxide and prostaglandins to reactive hyperemia in human forearm. Journal of Applied Physiology, 81(4), 1807-1814.

[17] Makino, T., Wakushima, H., Okamoto, T., Okukubo, Y., Saito, K. and Kano, Y. (2002) Effects of Kangen-karyu on coagulation system and platelet aggregation in mice. Biological \& Pharmaceutical Bulletin, 25(4), 523-525.

[18] Suzuki, Y., Goto, K., Ishige, A., Komatsu, Y. and Kamei, J. (1998) Effect of Gosha-jinki-gan, a Kampo medicine, on enhanced platelet aggregation in streptozotocin-induced 
diabetic rats. Japanese Journal of Pharmacology, 78(1), 87- 91 .

[19] Nakajima, S., Noguchi, T., Taka, T., Ueda, T., Kaizu, K., Fukamizu, M., Fujita, S., Tabuchi, M. and Yamamoto, J.
(2000) A global platelet test of thrombosis and thrombolysis detects a prothrombotic state in some patients with non-insulin dependent diabetes and in some patients with stroke. Platelets, 11, 459-466. 